\title{
YAP activates the Hippo pathway in a negative feedback loop
}

\author{
Xiaoming Dai ${ }^{1,}$, Huan Liu ${ }^{1,}$, Shuying Shen ${ }^{1,2}$, Xiaocan Guo ${ }^{1}$, Huan Yan ${ }^{1}$, Xinyan $\mathrm{Ji}^{1}$, Li Li ${ }^{3}$, Jun Huang ${ }^{1}$, \\ Xin-Hua Feng ${ }^{1}$, Bin Zhao ${ }^{1}$ \\ ${ }^{I}$ Life Sciences Institute and Innovation Center for Cell Signaling Network, Hangzhou, Zhejiang 310058, China; ${ }^{2}$ Institute of Bio- \\ chemistry, College of Life Science, Hangzhou, Zhejiang 310058, China; ${ }^{3}$ Institute of Aging Research, Hangzhou Normal Univer- \\ sity, Hangzhou, Zhejiang 311121, China
}

Cell Research (2017) 27:1073. doi:10.1038/cr.2017.96; published online 1 August 2017

Correction to: Cell Research (2015) 25:1175-1178. doi:10.1038/cr.2015.101; published online 28 August 2015

The authors apologized for the mistake of mis-labeling the affiliation for Drs Xiaoming Dai, Huan Liu, Shuying Shen, Xiaocan Guo, Huan Yan, Xinyan Ji, Jun Huang, Xin-Hua Feng, Bin Zhao. The correct form is as follows.
We apologize for any inconvenience that may have been caused by our error.

Xiaoming Dai ${ }^{1, *}$, Huan Liu ${ }^{1, *}$, Shuying Shen ${ }^{1,2}$, Xiaocan Guo ${ }^{1}$, Huan Yan ${ }^{1}$, Xinyan $\mathrm{Ji}^{1}$, Li Li ${ }^{3}$, Jun Huang ${ }^{1}$, Xin-Hua Feng ${ }^{1}$, Bin Zhao ${ }^{1}$

${ }^{1}$ Life Sciences Institute and Innovation Center for Cell Signaling Network, Zhejiang University, Hangzhou, Zhejiang 310058, China; ${ }^{2}$ Institute of Biochemistry, College of Life Sciences, Zhejiang University, Hangzhou, Zhejiang 310058, China; ${ }^{3}$ Institute of Aging Research, Hangzhou Normal University, Hangzhou, Zhejiang 311121, China

*These two authors contributed equally to this work. 